\title{
Codesigned Wideband High-Efficiency Filtering SIW Slot Antenna with High Selectivity and Flat Gain Response
}

\author{
Peng Kai Li $\mathbb{D},{ }^{1}$ Chang Jiang You $\mathbb{D},{ }^{1}$ Hong Fang Yu, ${ }^{1}$ Yu Jian Cheng $\mathbb{D},{ }^{2}$ Yuan Wang Yang, \\ and Jian Hua Deng ${ }^{3}$ \\ ${ }^{1}$ School of Information and Communication Engineering, University of Electronic Science and Technology of China, \\ Chengdu 611731, China \\ ${ }^{2}$ School of Electronic Science and Engineering, University of Electronic Science and Technology of China, Chengdu 611731, China \\ ${ }^{3}$ School of Information and Software Engineering, University of Electronic Science and Technology of China, Chengdu 611731, China
}

Correspondence should be addressed to Chang Jiang You; cjyou@uestc.edu.cn

Received 26 December 2017; Revised 1 March 2018; Accepted 15 April 2018; Published 2 July 2018

Academic Editor: Giorgio Montisci

Copyright (C) 2018 Peng Kai Li et al. This is an open access article distributed under the Creative Commons Attribution License, which permits unrestricted use, distribution, and reproduction in any medium, provided the original work is properly cited.

\begin{abstract}
A substrate integrated waveguide (SIW) slot antenna with wideband bandpass filtering performance is proposed in this journal. It consists of a SIW cavity, a transverse slot, three metal posts, and a SMA connector. Three metal posts split the SIW cavity into two $\mathrm{TE}_{110}$-mode resonators. A long nonresonant transverse slot is utilized to realize a half $\mathrm{TE}_{110}$-mode resonator and generate radiation simultaneously so as to reduce the size and release the use of extra radiator. Three in-band resonance poles and a radiation null at both out-of-bands are obtained. A prototype is fabricated and measured. Measured results demonstrate the proposed antenna is with a center frequency of $4.26 \mathrm{GHz}$, a fractional bandwidth of $9.1 \%$, a high efficiency of $93 \%$, and flat gain response and good skirt selectivity of $270 \mathrm{~dB} / \mathrm{GHz}$ and $330 \mathrm{~dB} / \mathrm{GHz}$ for the lower and upper out-of-band, respectively.
\end{abstract}

\section{Introduction}

Modern wireless communications demand the RF frontend system to be compact, lightweight, low cost, high efficiency, and multifunctional. In most of the RF front ends, the bandpass filter and the antenna are the key components whose performance will immediately affect the system performance. In the traditional systems, they are usually designed separately and connected by a $50 \Omega$ or $75 \Omega$ transmission line, which not only increases the volume but also maybe degrades in-band performance due to the mismatch and extra insertion loss caused by the interconnections [1]. Recently, a concept of filtering antenna has been proposed by integrating the bandpass filter and the antenna into a single component with filtering and radiating functions simultaneously. The proper integration of the antenna and filter turns out to be an efficient way to reduce the loss and enhance the efficiency of this functional block for a frontend system $[2,3]$.
Substrate integrated waveguide (SIW) technology has been effectively applied to design high-performance filters and antennas due to its low insertion loss [4-6]. Some filtering antennas based on SIW technology have been reported in [7-12]. In $[7,8]$, the antenna is planar and designed by cascading resonators and radiator. In [9-12], 3D configurations by placing the resonators under the radiator are applied. However, in reported works, they suffer from more insertion loss as they all need an extra radiator to cascade the filtering circuit and the filtering circuit is realized by full mode resonators which will lead to a large lossy circuit size. What is more, as they have no radiation null (transmission zero) in gain response or the radiation nulls are far away from the passband, the out-of-band skirt selectivity in the reported works is low.

To overcome these problems, a codesigned wideband filtering substrate integrated waveguide (SIW) slot antenna with high selectivity, high efficiency, and flat gain response is proposed in this journal. A long nonresonant transverse 
slot is utilized to realize a half mode resonator and generate radiation simultaneously so as to reduce the size and release the use of extra radiator. Three resonance poles are achieved to enhance the bandwidth and gain flatness. Two radiation nulls are introduced to increase the out-of-band skirt selectivity. Antenna work mechanism is explained, and the results and discussion are given.

\section{Antenna Configuration and Mechanism}

2.1. Configuration of the Proposed Antenna. Figure 1 shows the configuration of the proposed filtering antenna. The antenna is designed on a F4B-2 substrate with a thickness of $6 \mathrm{~mm}$, a relative permittivity of 2.485, and a loss tangent of 0.0018. It is composed of a SIW cavity with size $W \times L$, a transverse slot with size ws $\times$ ls etched on the top metal layer, three metal posts in the cavity and a SMA connector as feed. The metal posts are with a diameter of $d$ and space of xt. The antenna configuration is symmetrical about the $y$-axis. The antenna is designed and optimized at the centre frequency of $4.25 \mathrm{GHz}$. The detailed dimensions are as follows: $L=51$, $W=49, m=5.3, d=2.4, \mathrm{xt}=13.8, l_{1}=23, l_{2}=13, \mathrm{ls}=29.4$, ws $=2.65, a=1.6$, and $s=3.2$ (unit: $\mathrm{mm}$ ).

The design evolution of the proposed antenna is illustrated in Figure 2. Firstly, a transverse slot is etched on the upper metal layer of SIW with end shorted (Ant. 1). Then, three metal posts are introduced at the right side of the slot (Ant. 2). Finally, a SIW cavity is formed and a SMA connector is used as feed (Ant. 3). It should be mentioned that the slot is nonresonant at the work band and its length is more than half a guide wavelength. The reflection coefficient $S_{11}$ and peak realized gain of Ant. 1-3 are given in Figure 3. From Figure 3(a), it can be found that the proposed antenna Ant. 3 has three resonance poles $f_{r 1}$, $f_{r 2}$, and $f_{r 3}$. From Figure $3(\mathrm{~b})$, it can be found that Ant. 1 has only one radiation null in gain response while Ant. 2 and Ant. 3 have two radiation nulls at almost the same frequencies. These phenomena will be explained in detail in the following section.

2.2. Antenna Mechanism of Radiation Nulls. The configuration of a SIW transmission line with end shorted is shown in Figure 4(a). When it is excited by $\mathrm{TE}_{10}$ mode, the surface current $I$ is with standing wave distributions, as expressed by

$$
I=I_{\max } \cos [\beta(f) z] .
$$

Here, $I_{\max }$ is defined as the maximum current magnitude, $\beta(f)$ denotes the phase constant at frequency $f$, and $z$ means the distance from the shorted end. Figure 4(b) gives the surface current distribution at two different frequency $f_{1}$ and $f_{2}$, which are labelled as case 1 and case 2, respectively. If a slot is etched on the top layer, it will generate radiation or not which depends on the current distribution at the position of the slot. It should be stated that there will be no radiation at the current null. Thus, if the slot is located at the position of current null as case 1 in Figure 4(b), there will be no radiation generated from the slot. And if the slot is not located at the position of current null as case 2 , there will be radiation generated from the slot. Hence, a radiation null will occur at the frequency where the current at the slot satisfies the following condition that

$$
I_{A A^{\prime}}=0 \text {. }
$$

That is the reason why one radiation null is generated in Ant. 1, which is also explained in [13].

Actually in Ant. 2 and Ant. 3, when three metal posts are introduced, a resonator $R_{1}$ is formed. The configuration of SIW transmission line loaded by a resonator and its equivalent circuit model is illustrated in Figure 5(a). The resonator $R_{1}$ can be simply modelled as shunt inductor and capacitor, and it resonates at $4.25 \mathrm{GHz}$. The phase shift at the reference plane in the one end shorted structure is twice that of a twoport resonator model as discussed in [14]. Thus, as shown in Figure 5(b), the phase shift $\varphi_{1}$ of the end shorted resonator $R_{1}$ can be expressed as

$$
\begin{aligned}
& \varphi_{1} \approx+180^{\circ} \text { (below resonance) }, \\
& \varphi_{1} \approx-180^{\circ} \text { (above resonance). }
\end{aligned}
$$

For Ant. 2 and Ant. 3, the resonator $R_{1}$ can be considered as the load of the transmission line, as illustrated in Figure 6(a). The phase difference $\varphi$ at the reference plane can be written as

$$
\varphi=2 \beta(f) l_{2}+\varphi_{1} .
$$

Here, $l_{2}$ denotes the distance from the three metal posts. According to transmission line theory [15], the current strength at the reference plane can be expressed as

$$
\left|I\left(z=-l_{2}\right)\right|=\left|\frac{V_{0}^{+}}{Z_{0}}\right||1-| \Gamma\left|e^{-j \varphi}\right| .
$$

Here, $V_{0}^{+}$means the voltage of incident wave, $Z_{0}$ denotes the characteristic impedance of the transmission line, and $\Gamma$ is voltage reflection coefficient. According to (5), the minimum current strength (current null) satisfies the condition that

$$
\varphi=2 n \pi, \quad n=0,1,2 \ldots .
$$

According to (3), (4), and (6), when current null occurs, the minimum $l_{2}$ should meet that

$$
2 \beta(f) l_{2} \approx \pi
$$

It means when $l_{2}$ is about $\lambda_{\mathrm{g}} / 4$ ( $\lambda_{\mathrm{g}}$ is guide wavelength), two current nulls will occur at both sides of the resonance. Figure 6(b) gives the phase difference when $l_{2}$ changes near $\lambda_{\mathrm{g}} / 4(13 \mathrm{~mm})$. The phase difference is modularized by $2 \pi$ and is limited to the $\left[-180^{\circ}\right.$ and $\left.180^{\circ}\right]$ range. It can be found when $\varphi=0$, there exist two frequency $f_{n 1}$ and $f_{n 2}$ at both sides of $4.25 \mathrm{GHz}$. From the abovementioned analysis, the frequency where $\varphi=0$ is the frequency of current null. It can also be seen from Figure 6(b), frequencies of the two current nulls shift downward when $l_{2}$ increases from $11 \mathrm{~mm}$ to $15 \mathrm{~mm}$. It means the upper current null will come close to the resonance, and the lower current null will be away from the resonance. 


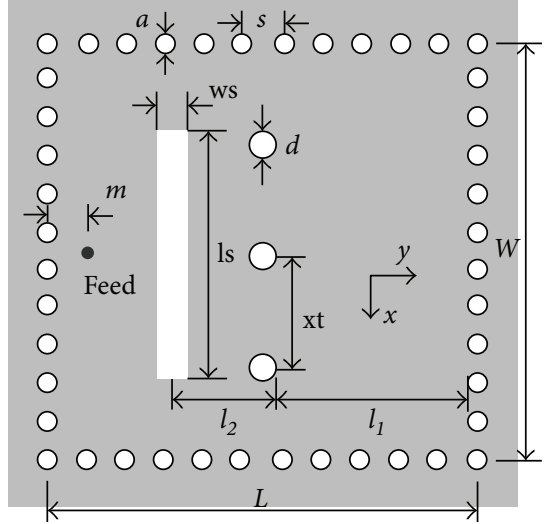

(a)

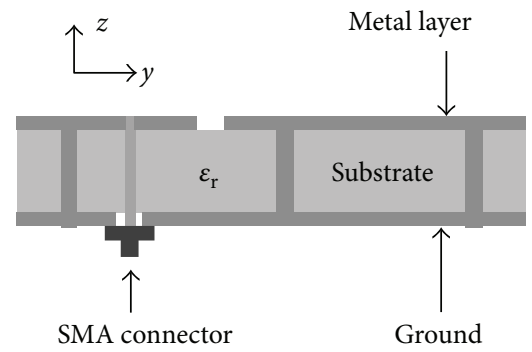

(b)

Figure 1: Configuration of the proposed filtering antenna. (a) Top view. (b) Side view.

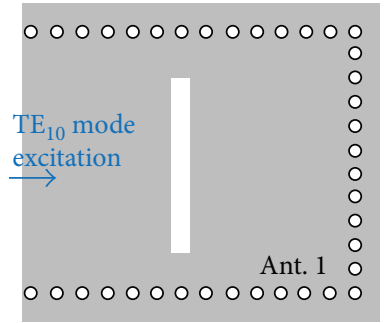

(a)

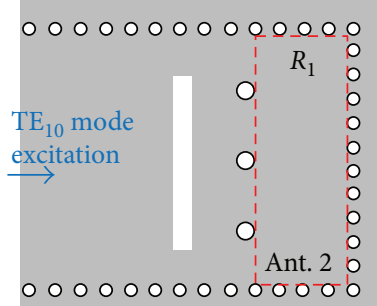

(b)

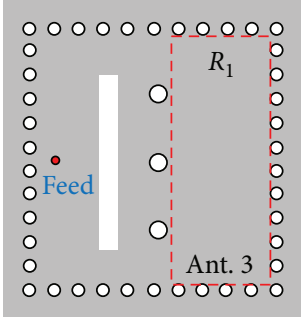

(c)

FIGURE 2: Design evolution. (a) SIW transverse slot. (b) SIW transverse slot with metal posts. (c) SIW cavity-backed transverse slot with metal posts.

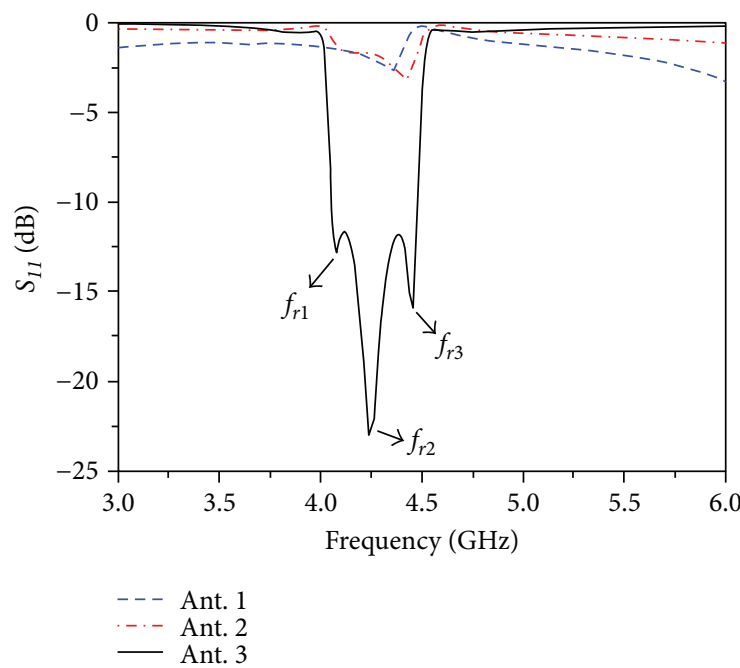

(a)

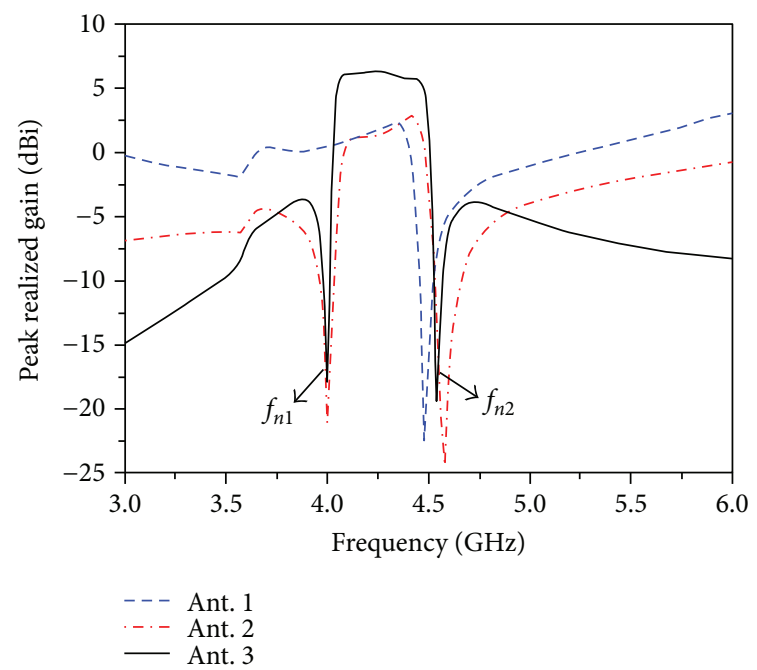

(b)

Figure 3: Simulated results of Ant. 1, Ant. 2, and Ant. 3. (a) Reflection coefficient $S_{11}$. (b) Peak realized gain.

According to transmission line theory, input impedance is defined as $Z_{\text {in }}=V(z) / I(z)$. When the current is minimum, the input impedance is maximum. So the current null can be deduced from the feature of input impedance. Figure 7 gives the simulated magnitude of input impedance $Z_{A A^{\prime}}$ with the variation of $l_{2}$. It can be found that one impedance peak is observed at both sides of $4.25 \mathrm{GHz}$. The frequency of the impedance peak is the frequency of current null. Due to the lossy material used in the simulation, when approaching the resonance, the loss is more and voltage reflection 


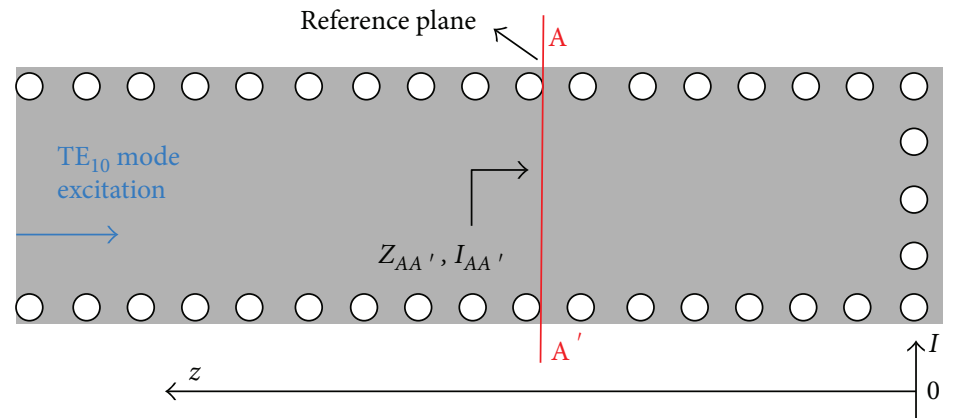

(a)

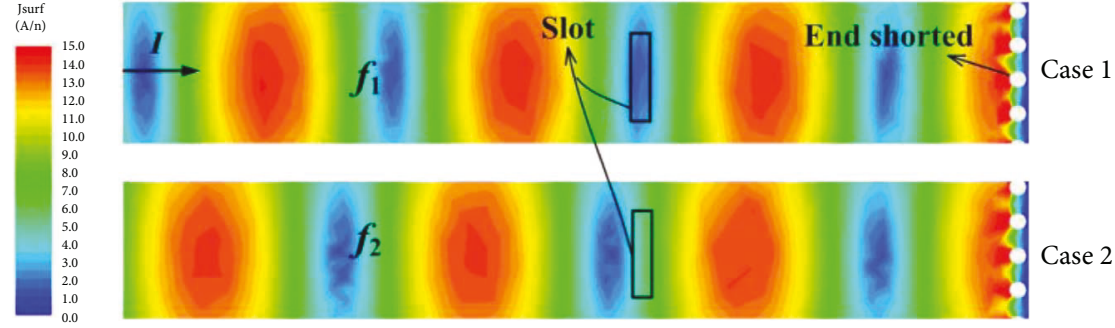

(b)

FIGURE 4: SIW transmission line with end shorted and surface current distributions. (a) Configuration of SIW transmission line with end shorted. (b) Surface current distributions at different frequencies.

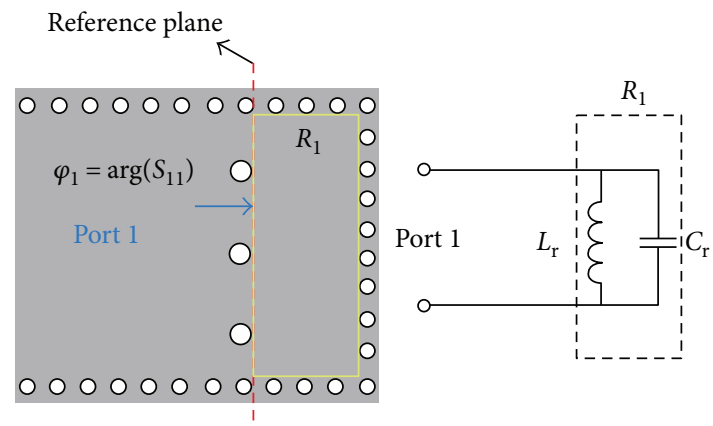

(a)

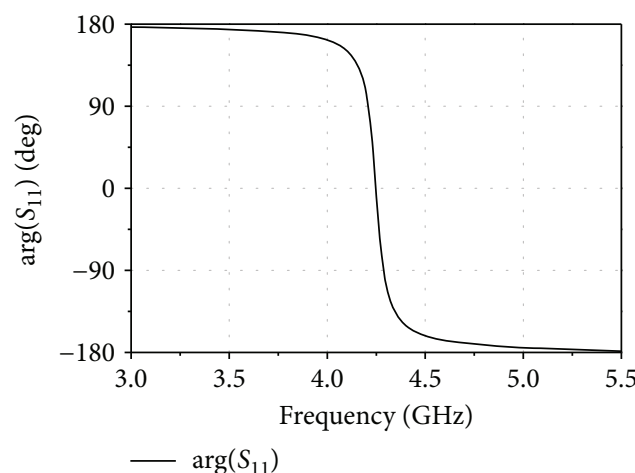

(b)

FIGURE 5: Illustration of analysis model and the results. (a) Analysis model of an end shorted resonator. (b) Simulated phase of $S_{11}$.

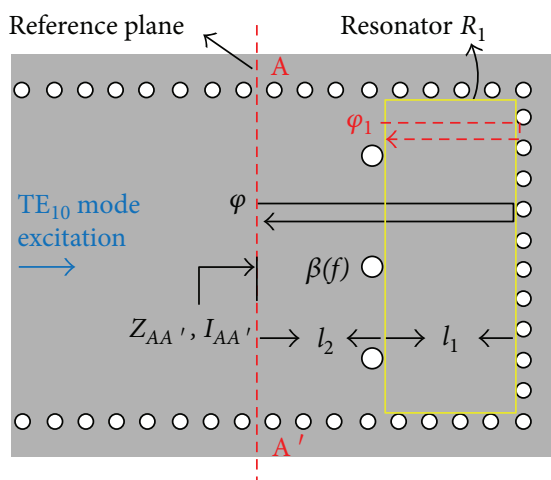

(a)

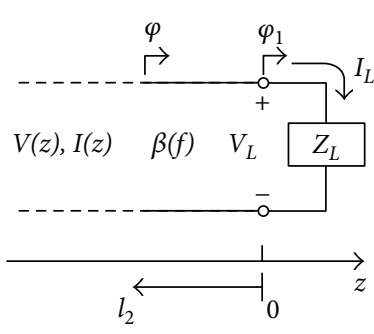

$--l_{2}=11 \mathrm{~mm}$

(b)

FIGURE 6: Illustration of analysis model and the results. (a) Analysis model of Ant. 2. (b) Simulated phase difference. 
coefficient $\Gamma$ becomes smaller. Meanwhile, the minimum current strength becomes larger according to (5). It means the impedance peak becomes smaller near the resonance $4.25 \mathrm{GHz}$ as can be seen in Figure 7. So the depth of the two radiation nulls in Ant. 2 and Ant. 3 can be estimated from Figure 7. A larger impedance peak means a deeper radiation null, as can be proved from the simulated peak realized gain of Ant. 3 with different $l_{2}$ in Figure 8.

To achieve two symmetrical radiation nulls about $4.25 \mathrm{GHz}$, here in the proposed antenna, the slot is located at the position where $l_{2}$ is chosen to be $13 \mathrm{~mm}$ which is about a quarter phase wavelength at the resonant frequency of $R_{1}$.

2.3. Antenna Mechanism of Resonance Poles. As can be seen in Figure 3(a), three resonance poles $f_{r 1}, f_{r 2}$, and $f_{r 3}$ are generated in Ant. 3. Actually, when three metal posts are introduced, the large cavity is divided into two $\mathrm{TE}_{110}$-mode resonators. What's more, when a long slot is cut, a half $\mathrm{TE}_{110}$-mode resonator is created. The three resonant poles are generated by the couplings between the three resonators, and they can be explained by the basic resonant mode superposition. Figure 9 gives the simulated $\mathrm{H}$-filed at different phase of the three resonance poles and their superposition modes. The modes created by the two $\mathrm{TE}_{110}$-mode resonators and their coupling can be considered as an even mode and an odd mode [16]. Thus, as illustrated in Figure 9(a), the first resonance pole $f_{r 1}$ can be considered as the superposition of a half $\mathrm{TE}_{110}$ mode and an even mode. As illustrated in Figure 9(b), the second resonance pole $f_{r 2}$ can be considered as the superposition of a half $\mathrm{TE}_{110}$ mode and a $\mathrm{TE}_{110}$ mode. As illustrated in Figure 9(c), the third resonance pole $f_{r 3}$ can be considered as the superposition of a half $\mathrm{TE}_{110}$ mode and an odd mode. It should be noticed that all the resonance poles are in relation to the half $\mathrm{TE}_{110}$ mode. This is because only the half $\mathrm{TE}_{110}$ mode can generate radiation through the slot, while at even and odd mode, the slot is almost located at the position of current null. It is innovative that in the proposed design, the slot not only acts as a radiator but also is essential to the formation of the half mode resonator. This merged structure is different to the commonly used method which will introduce extra radiator.

\section{Results and Discussion}

The proposed antenna is fabricated and measured, and the photograph is shown in Figure 10. The simulated and measured reflection coefficient $S_{11}$, realized gains, and total efficiencies are illustrated in Figure 11. It can be found that measured bandwidth $\left(S_{11}<-10 \mathrm{~dB}\right)$ is 9.1\% (4.09$4.48 \mathrm{GHz}$ ), agreeing well with the simulated one of $10.1 \%$ (4.05-4.48 GHz).

The measured and simulated average realized gains over the work band are $6.2 \mathrm{dBi}$ and $6.3 \mathrm{dBi}$, respectively. The gain response is flat over the work band with ripples less than $0.4 \mathrm{~dB}$, which is attributed to the nonresonant slot radiation. The bandpass filtering performance is remarkable, as the efficiency over the work band reaches to a maximum as high as $93 \%$ which means insertion loss of about only $0.3 \mathrm{~dB}$ is introduced, while the out-of-band efficiency approaches zero. The
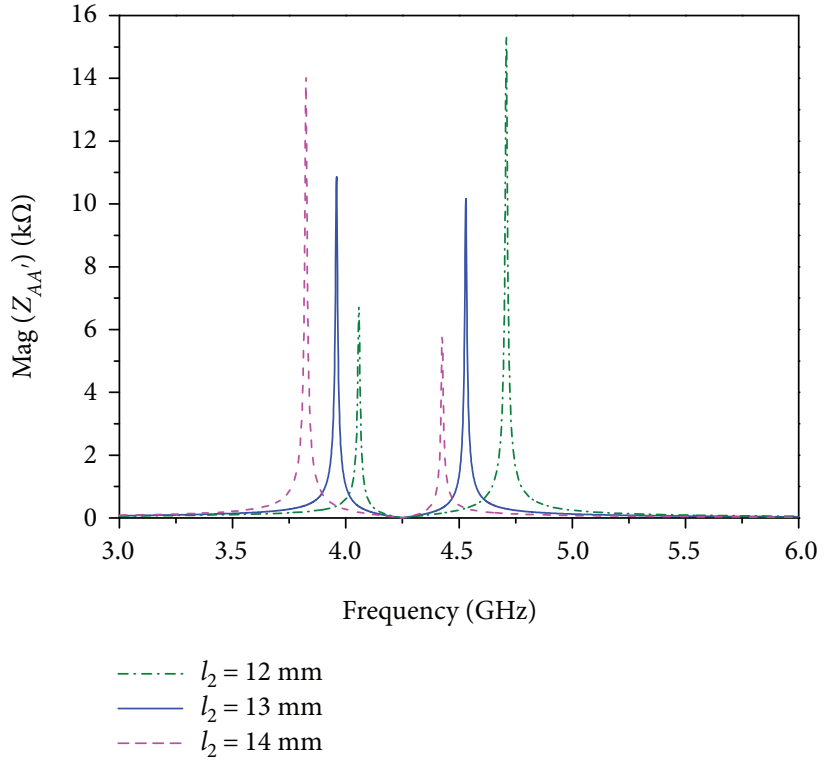

Figure 7: Simulated magnitude of $Z_{A A^{\prime}}$ illustrated in Figure 5(a).

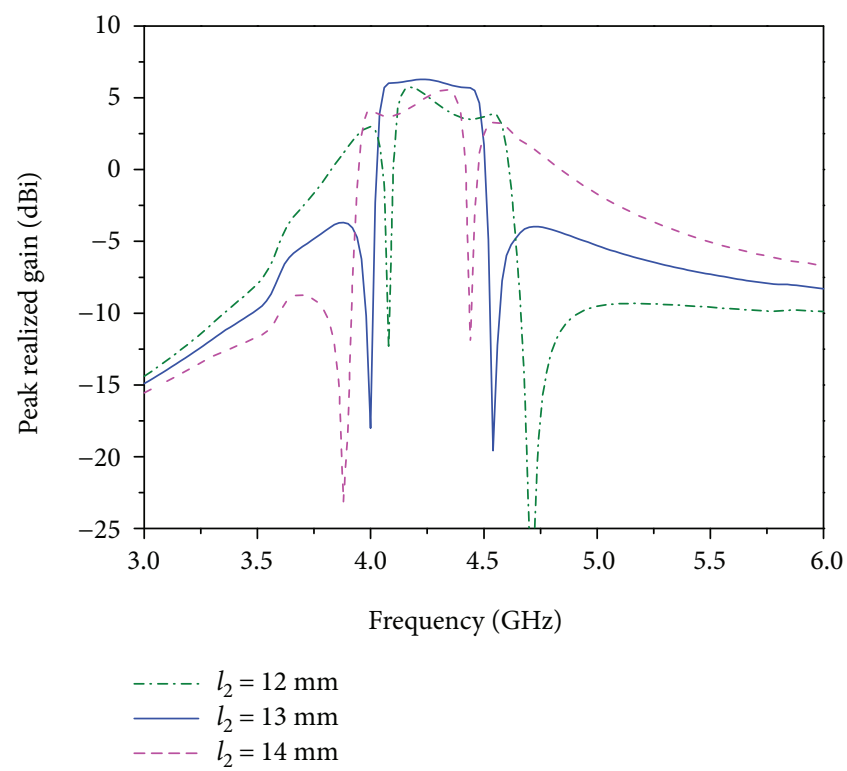

Figure 8: Simulated peak realized gain of Ant. 3 with different $l_{2}$.

high efficiency is benefited from the nonresonant slot radiation and small lossy circuit area as a half mode resonator is realized and no extra radiator is utilized. As can be observed in Figure 11, two measured radiation nulls at $4.04 \mathrm{GHz}$ and $4.56 \mathrm{GHz}$ in gain response are very close to the pass-band, so high skirt selectivity is achieved. The measured lower and upper out-of-band skirt selectivity is of $270 \mathrm{~dB} / \mathrm{GHz}$ and $330 \mathrm{~dB} / \mathrm{GHz}$, respectively.

The simulated and measured radiation patterns at the three resonance poles $4.12 \mathrm{GHz}, 4.26 \mathrm{GHz}$, and $4.46 \mathrm{GHz}$ are shown in Figure 12. It can be seen almost symmetrical radiation patterns are observed in $\mathrm{E} / \mathrm{H}$ plane. The slightly tilts at E-plane patterns are mainly caused by the offcentered slot position. Good front-to-back ratios and cross 

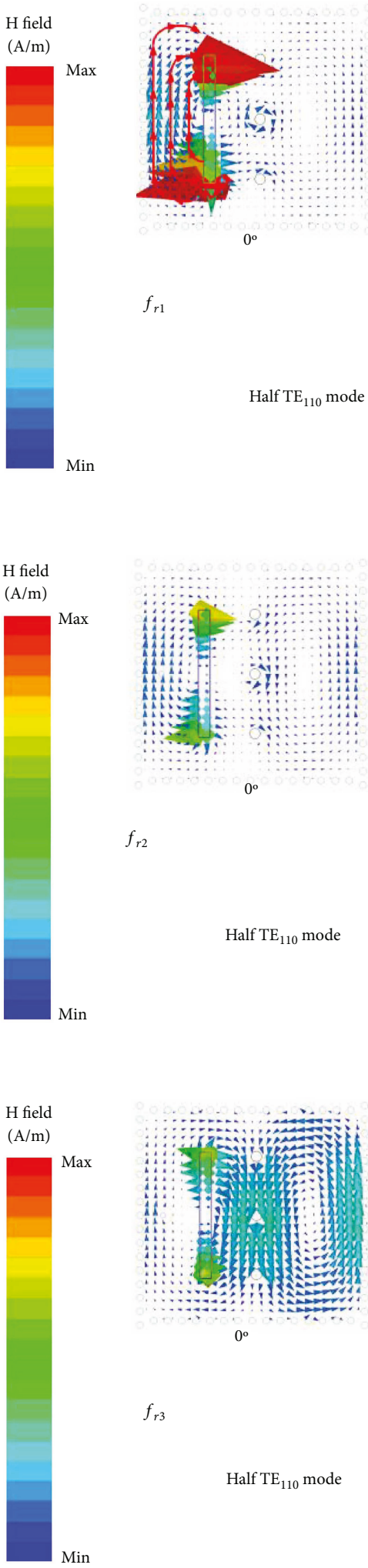

$f_{r 2}$

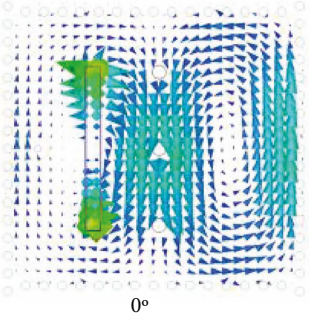

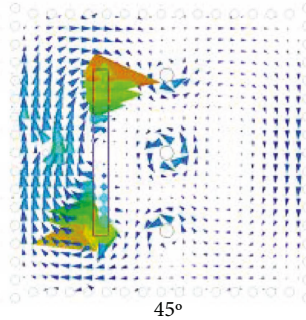
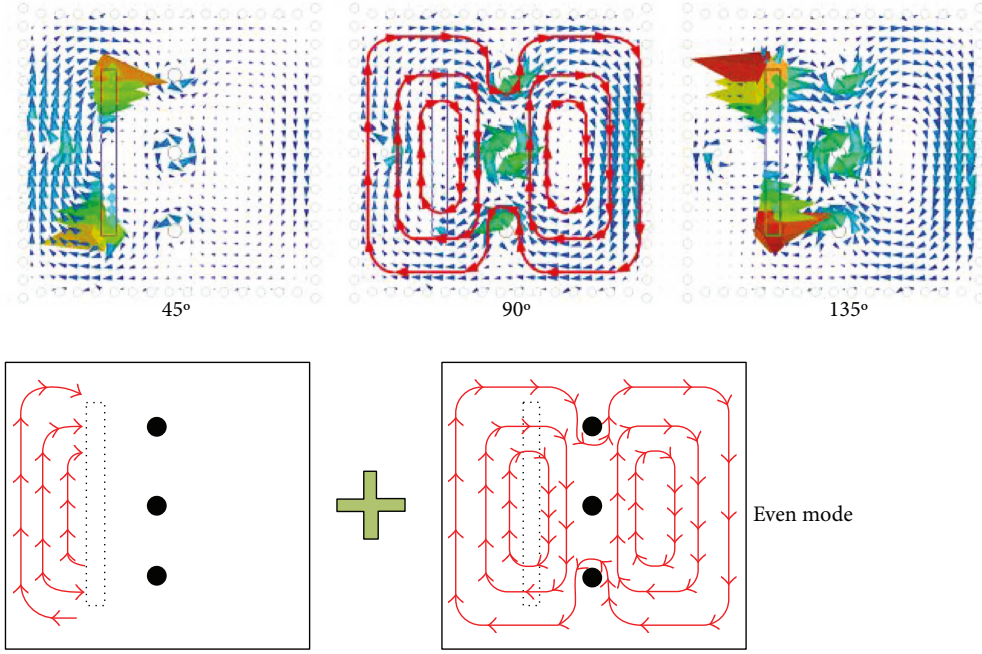

(a)
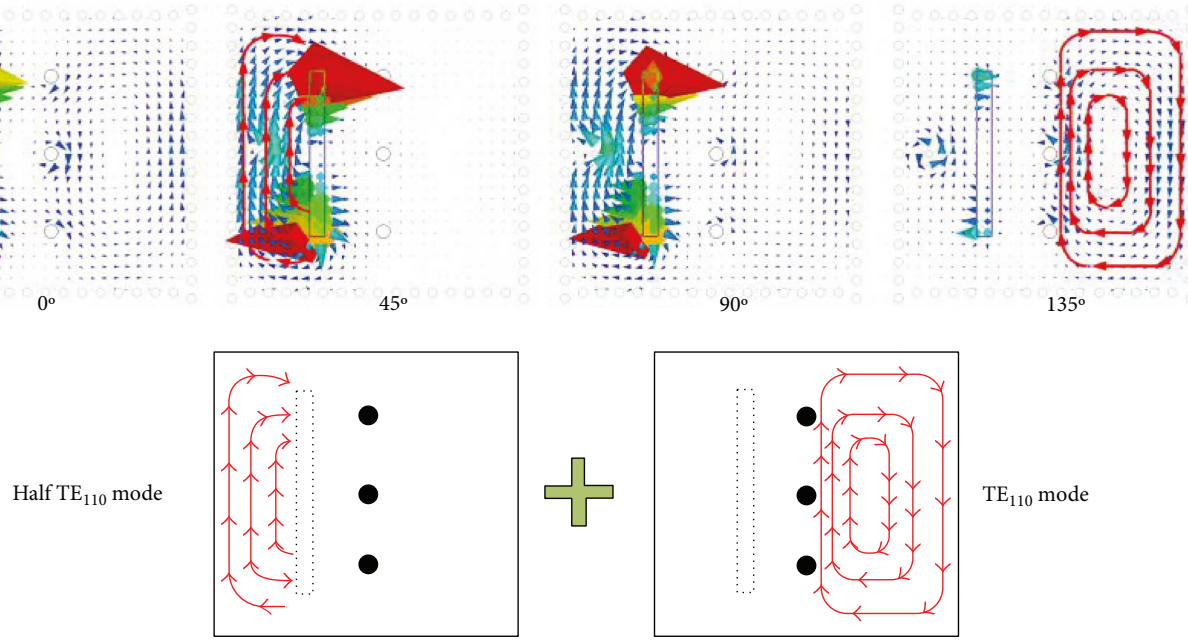

(b)
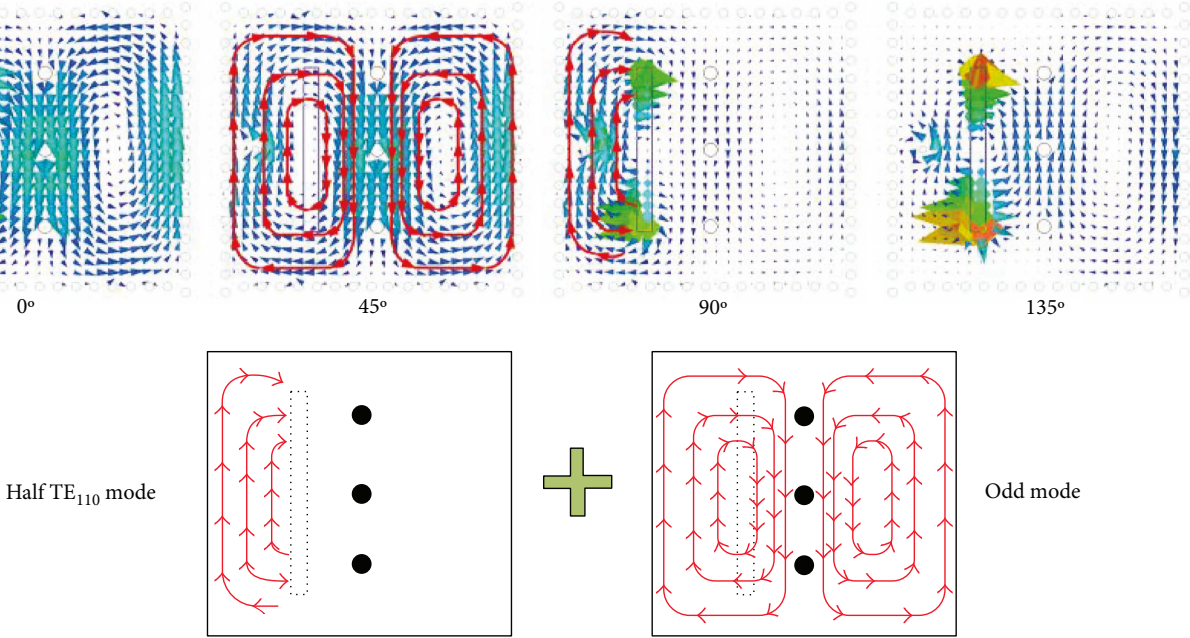

(c)

FIGURE 9: Illustration of the mechanism of resonance poles in Ant. 3. (a) First resonance pole $f_{r 1}$. (b) Second resonance pole $f_{r 2}$. (c) Third resonance pole $f_{r 3}$. 


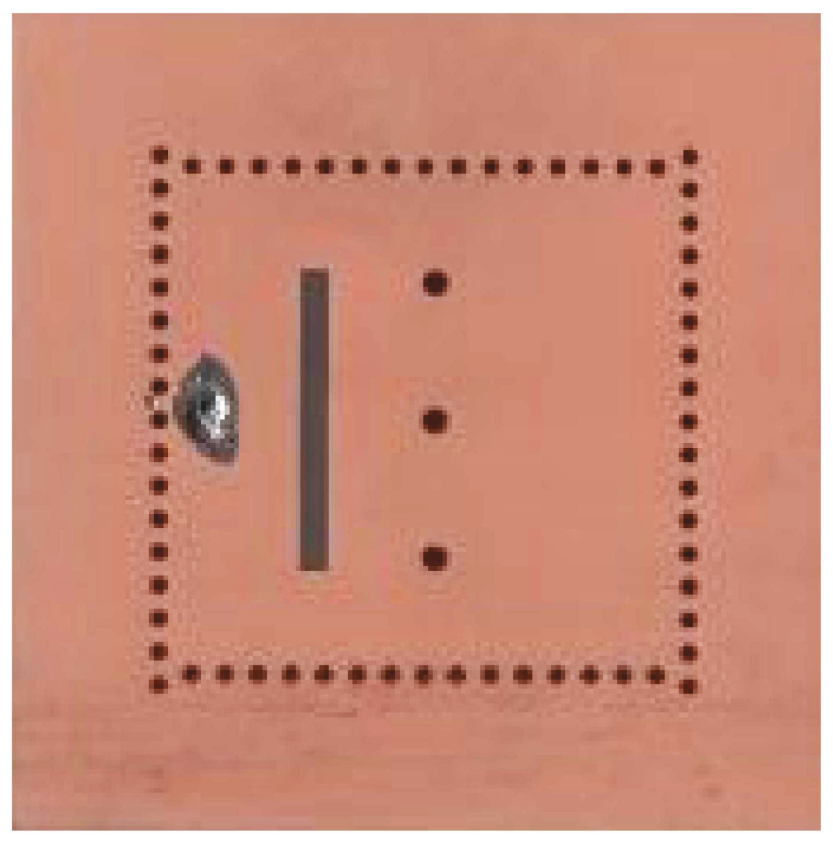

Figure 10: Photograph of the fabricated antenna.

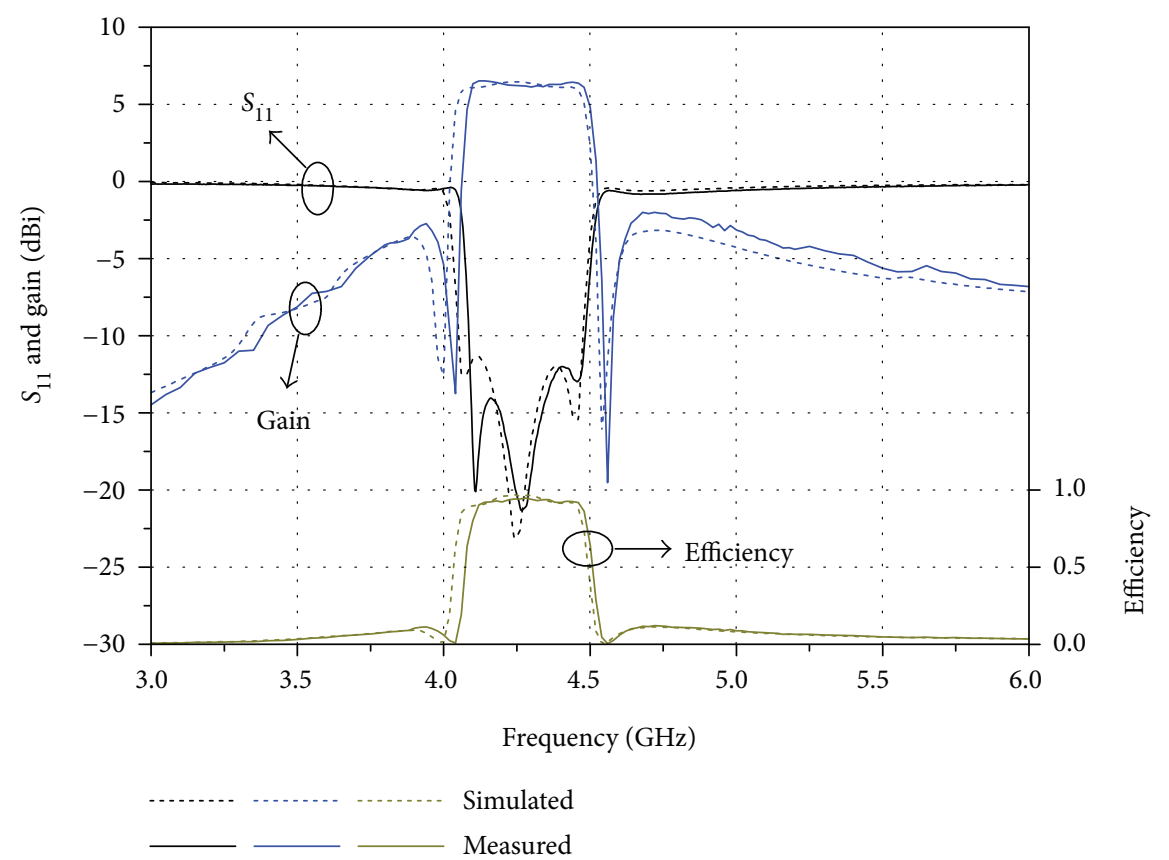

Figure 11: Simulated (dashed lines) and measured (solid lines) reflection coefficient $S_{11}$, realized gains, and total efficiencies.

polarization levels of more than $20 \mathrm{~dB}$ are obtained at both planes. The simulated copolarization patterns are in accordance with the measured ones, and the copolarization patterns are stable over the work band. The discrepancy in cross polarization between simulated and measured value is mainly attributed to the slightly tilted antenna placement during measurement.
A comprehensive comparison with previous works utilizing SIW slot is summarized in Table 1. It can be found in our work the advantages of wideband, high selectivity, and high efficiency (Eff.) compared to other works. The high efficiency can also be deduced from the lossy circuit area which can be evaluated by size $\times$ layer. It is obvious that our work has the least value of size $\times$ layer. 

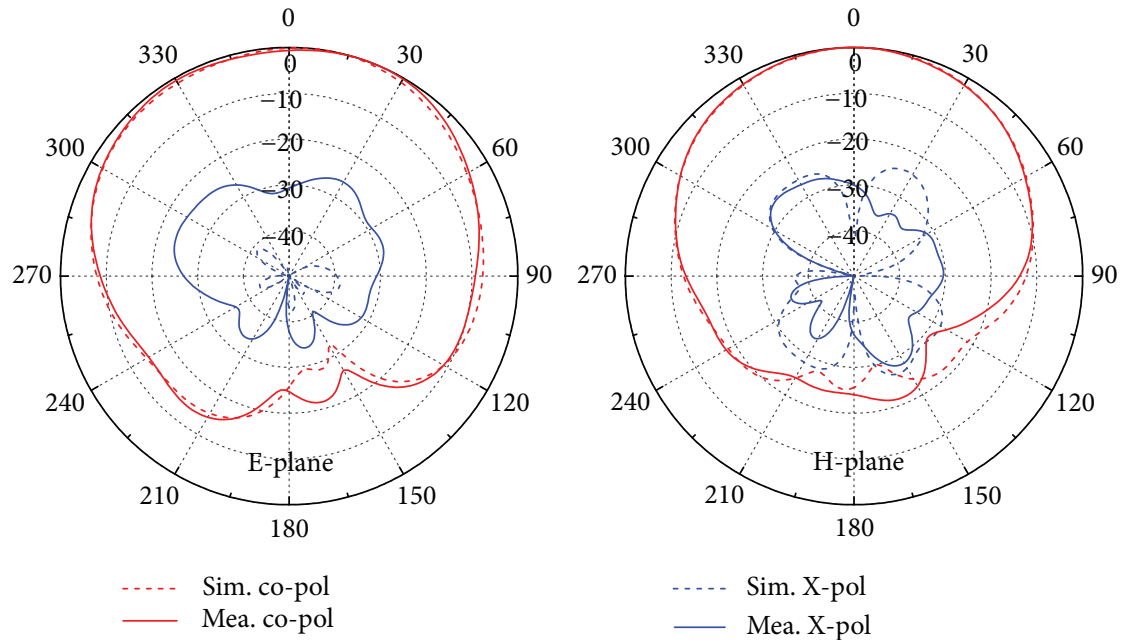

(a)
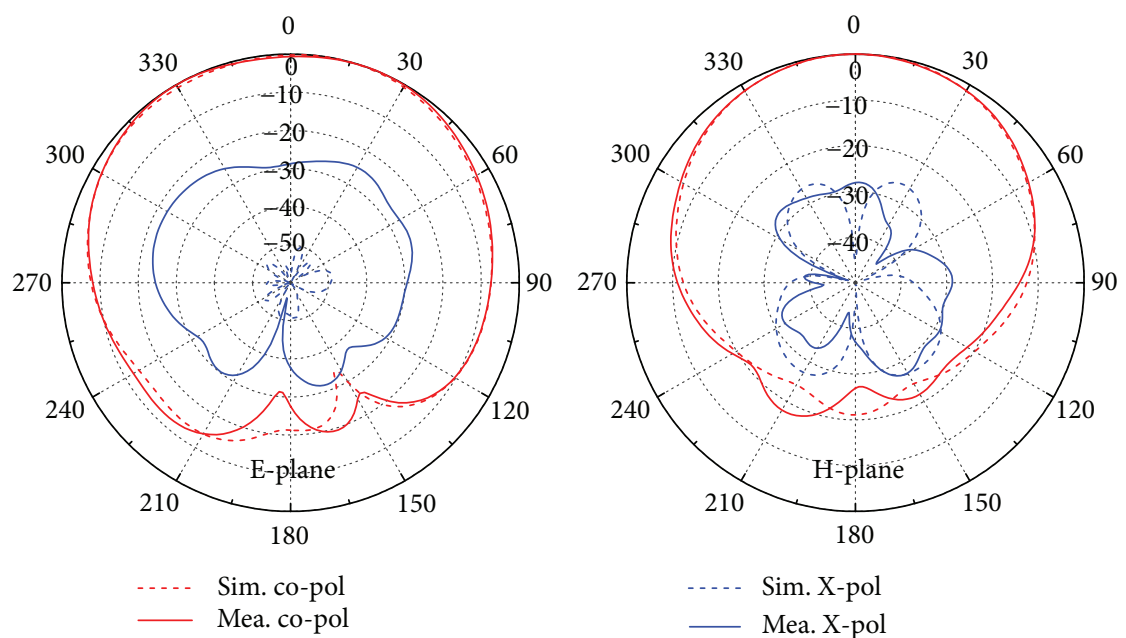

(b)
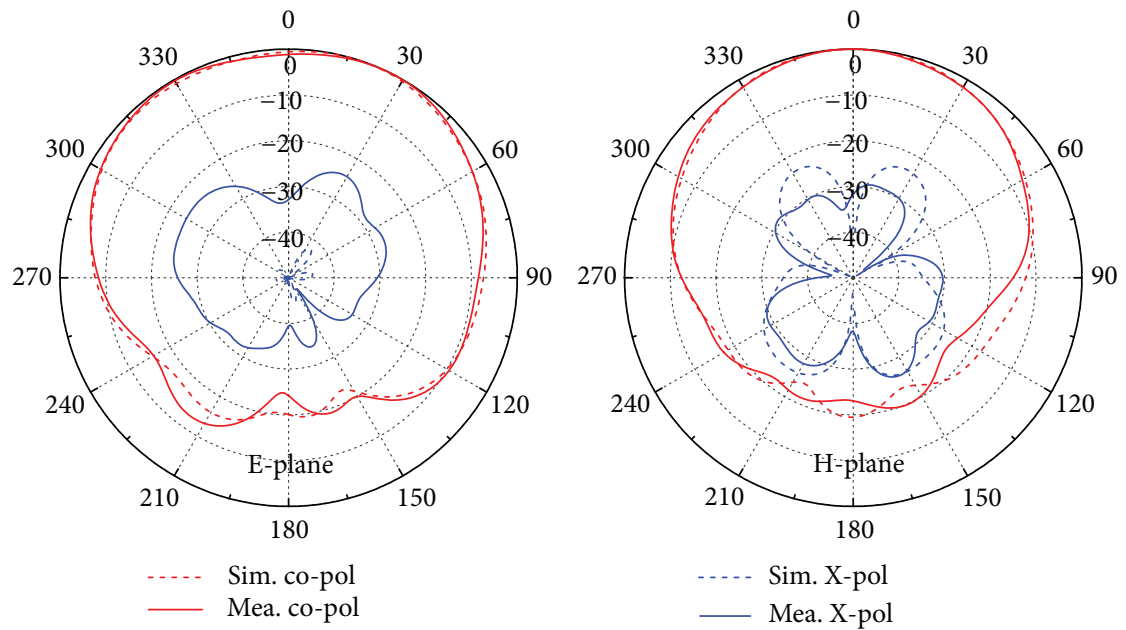

(c)

FIGURE 12: Simulated and measured radiation patterns at three resonance poles. (a) $4.12 \mathrm{GHz}$. (b) $4.26 \mathrm{GHz}$. (c) $4.46 \mathrm{GHz}$. 
TABLE 1: Comparisons with previous reported works.

\begin{tabular}{lcccccc}
\hline Ref & Number of Pole & Number of Null & BW $(\%)$ & Selectivity $(\mathrm{dB} / \mathrm{GHz})$ & Eff. $(\%)$ & Size $\left(\lambda_{\mathrm{g}} \times \lambda_{\mathrm{g}}\right)$ \\
\hline$[8]$ & 4 & 0 & 6 & $113 / 74$ & 89 & $1.3 \times 1.7$ \\
{$[9]$} & 2 & 0 & 3 & $63 / 28$ & 58.9 & $0.9 \times 0.9$ \\
{$[10]$} & 3 & 2 & 1.6 & $96 / 55$ & 86 & $0.7 \times 0.9$ \\
{$[11]$} & 3 & 0 & 5.5 & $26 / 57$ & N.A. & $1.4 \times 1.6$ \\
Ours & 3 & $\mathbf{2}$ & $\mathbf{9 . 1}$ & $\mathbf{2 7 0 / 3 3 0}$ & $\mathbf{9 3}$ & $\mathbf{1 . 1} \times \mathbf{1 . 2}$ \\
\hline
\end{tabular}

$\lambda_{g}$ is the guide wavelength at the centre frequency of the work band. Selectivity is calculated as $\left|\alpha_{\max }-\alpha_{\min }\right| /\left|f_{z}-f_{c}\right|$, where $\alpha_{\max }$ is the attenuation of the first out-of-band radiation null or $20 \mathrm{~dB}$ attenuation if no radiation null and $\alpha_{\min }$ is the $3 \mathrm{~dB}$ attenuation; $f_{\mathrm{z}}$ and $f_{\mathrm{c}}$ are their corresponding frequency.

\section{Conclusion}

A substrate integrated waveguide slot antenna with bandpass filtering performance in gain response is presented in this journal. A long nonresonant slot is introduced to realize a half mode resonator and generate radiation so as to reduce the size and release the use of extra radiator. Two radiation nulls are generated to enhance the selectivity. The measured results show that wide bandwidth of $9.1 \%$, high selectivity of $270 / 330 \mathrm{~dB} / \mathrm{GHz}$ for lower/upper out-of-band, and high efficiency of $93 \%$ and flat gain response are obtained in the proposed antenna. The high performance indicates it is a good candidate for the functional module integrated with filter and antenna in RF front system.

\section{Conflicts of Interest}

The authors declare that they have no competing interests.

\section{Acknowledgments}

This work was supported by Fundamental Research Funds for the Central Universities under Grant ZYGX2016J006.

\section{References}

[1] X. Y. Zhang, W. Duan, and Y.-M. Pan, "High-gain filtering patch antenna without extra circuit," IEEE Transactions on Antennas and Propagation, vol. 63, no. 12, pp. 5883-5888, 2015.

[2] W. J. Wu, Y. Z. Yin, J. J. Xie, X. S. Ren, and S. L. Zuo, “Low-cost microstrip filter antenna with a monopole-like radiation pattern for RF front end," Microwave and Optical Technology Letters, vol. 54, no. 8, pp. 1810-1814, 2012.

[3] S. Chen, Y. Zhao, M. Peng, and Y. Wang, "A codesigned compact dual-band filtering antenna with PIN loaded for WLAN applications," International Journal of Antennas and Propagation, vol. 2014, Article ID 826171, 6 pages, 2014.

[4] X. H. Zhang, G. Q. Luo, and L. X. Dong, "Substrate integrated waveguide fed cavity backed slot antenna for circularly polarized application," International Journal of Antennas and Propagation, vol. 2013, Article ID 316208, 6 pages, 2013.

[5] G. Q. Luo, Z. F. Hu, L. X. Dong, and L. L. Sun, "Planar slot antenna backed by substrate integrated waveguide cavity," IEEE Antennas and Wireless Propagation Letters, vol. 7, pp. 236-239, 2008.

[6] J. Wang and Y. J. Cheng, "W-band hybrid unequal feeding network of waveguide and substrate integrated waveguide for high efficiency and low sidelobe level slot array antenna application," International Journal of Antennas and Propagation, vol. 2017, Article ID 7183434, 8 pages, 2017.

[7] Y. Yusuf and X. Gong, "Integration of three-dimensional high$Q$ filters with aperture antennas and bandwidth enhancement utilising surface waves," IET Microwaves, Antennas and Propagation, vol. 7, no. 7, pp. 468-475, 2013.

[8] Y. Yusuf and X. Gong, "Compact low-loss integration of highQ 3-D filters with highly efficient antennas," IEEE Transactions on Microwave Theory and Techniques, vol. 59, no. 4, pp. 857-865, 2011.

[9] Y. Yusuf, H. Cheng, and X. Gong, "A seamless integration of 3D vertical filters with highly efficient slot antennas," IEEE Transactions on Antennas and Propagation, vol. 59, no. 11, pp. 4016-4022, 2011.

[10] H. Cheng, Y. Yusuf, and X. Gong, "Vertically integrated threepole filter/antennas for array applications," IEEE Antennas and Wireless Propagation Letters, vol. 10, pp. 278-281, 2011.

[11] H. Chu, C. Jin, J.-X. Chen, and Y.-X. Guo, "A 3-D millimeter-wave filtering antenna with high selectivity and low cross-polarization," IEEE Transactions on Antennas and Propagation, vol. 63, no. 5, pp. 2375-2380, 2015.

[12] H. Chu, J.-X. Chen, S. Luo, and Y.-X. Guo, "A millimeter-wave filtering monopulse antenna array based on substrate integrated waveguide technology," IEEE Transactions on Antennas and Propagation, vol. 64, no. 1, pp. 316-321, 2016.

[13] P. K. Li, C. J. You, H. F. Yu, X. Li, Y. W. Yang, and J. H. Deng, "Codesigned high-efficiency single-layered substrate integrated waveguide filtering antenna with a controllable radiation null," IEEE Antennas and Wireless Propagation Letters, vol. 17, no. 2, pp. 295-298, 2018.

[14] J. B. Thomas, "Cross-coupling in coaxial cavity filters - a tutorial overview," IEEE Transactions on Microwave Theory and Techniques, vol. 51, no. 4, pp. 1368-1376, 2003.

[15] D. M. Pozar, Microwave Engineering, John Wiley \& Sons, Inc., Hoboken, NJ, USA, Third edition, 2005.

[16] J.-S. Hong and M. J. Lancaster, Microstrip Filters for RF/Microwave Applications, John Wiley \& Sons, Inc., New York, NY, USA, 2001. 


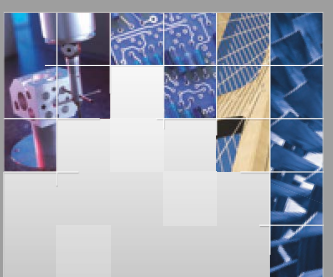

\section{Enfincering}
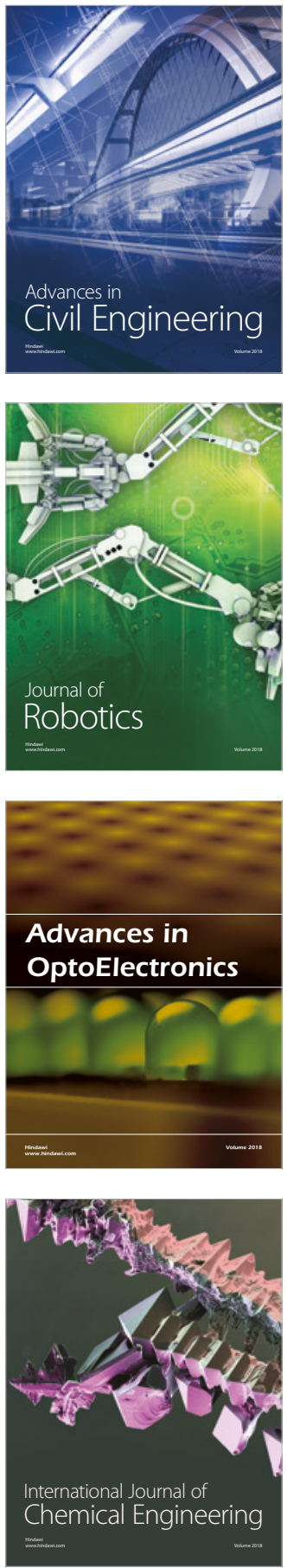

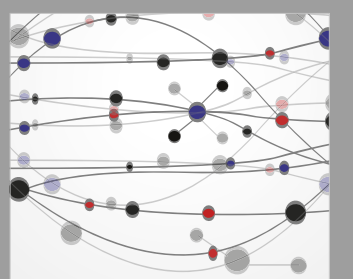

\section{Rotating \\ Machinery}

The Scientific World Journal

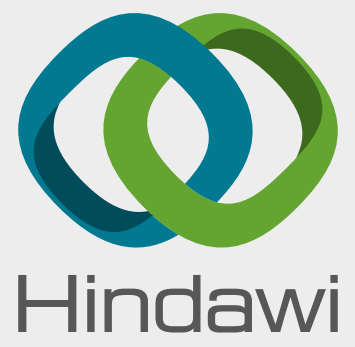

Submit your manuscripts at

www.hindawi.com
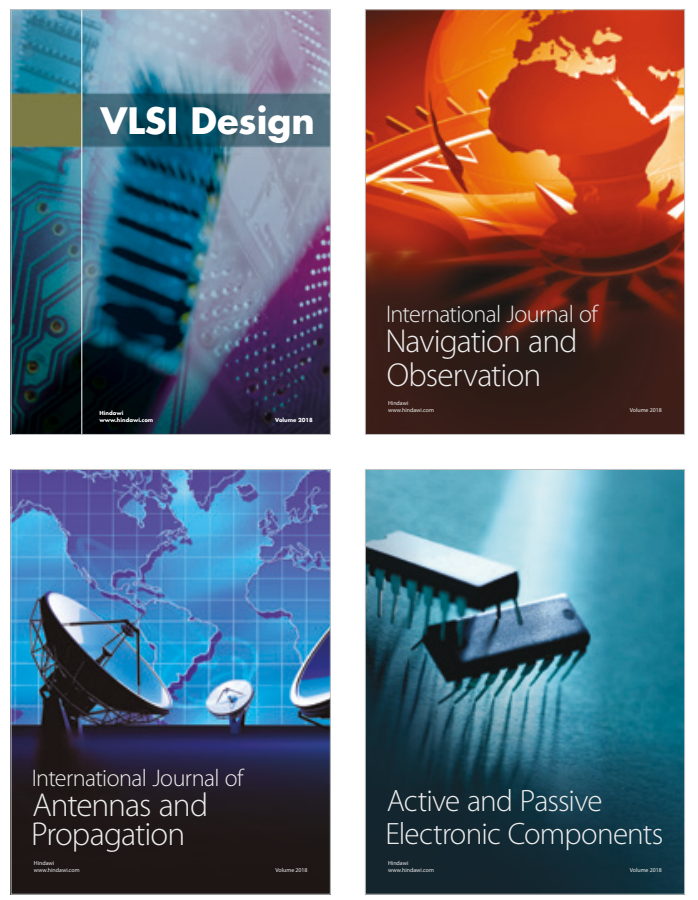
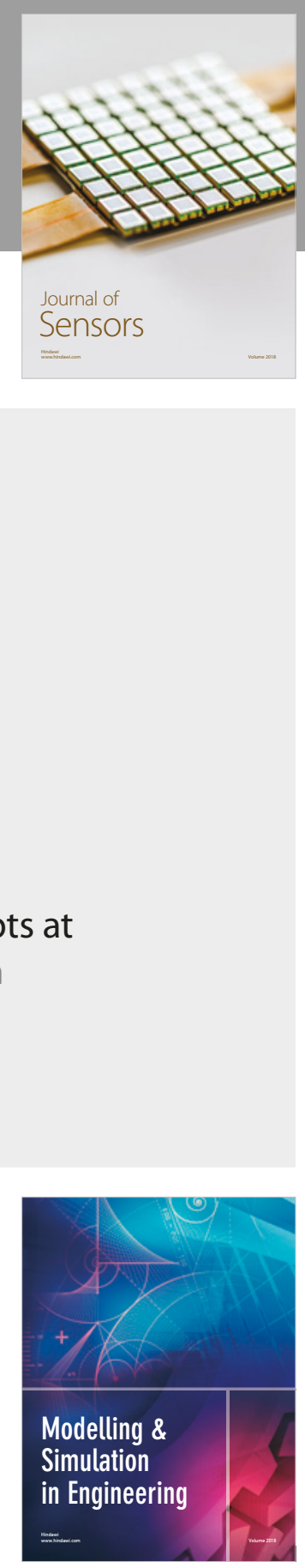

\section{Advances \\ Multimedia}
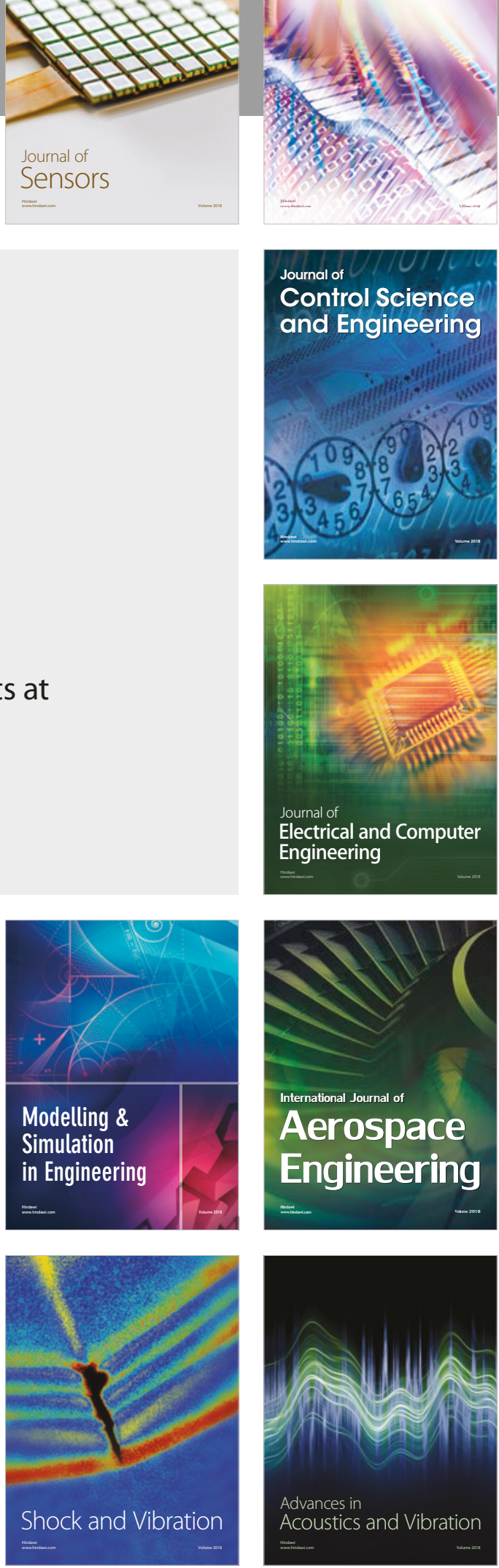